\title{
Thioacylation reactions for the surface functionalization of phosphorus-containing dendrimers
}

\author{
Patrice Marchand, ${ }^{\mathrm{a}}$ Laurent Griffe, ${ }^{\mathrm{a}}$ Anne-Marie Caminade, ${ }^{\mathrm{a}} *$ Jean-Pierre Majoral, ${ }^{\mathrm{a}} *$ \\ Mathias Destarac, ${ }^{\mathrm{b}}$ Frédéric Leising ${ }^{\mathrm{b}}$
}

${ }^{a}$ Laboratoire de Chimie de Coordination du CNRS, 205 route de Narbonne, 31077 Toulouse Cedex 4, France

${ }^{b}$ Rhodia, Centre de Recherches d'Aubervilliers, 52 rue de la Haie Coq, 93308 Aubervilliers Cedex, France

caminade@lcc-toulouse.fr, majoral@lcc-toulouse.fr

\section{Supporting Information}

General. All manipulations were carried out with standard high vacuum and dry-argon techniques. The solvents were freshly dried and distilled (THF and ether over sodium/benzophenone, pentane and $\mathrm{CH}_{2} \mathrm{Cl}_{2}$ over phosphorus pentoxide, toluene over sodium), they were degassed prior to use in the case of experiments using tricoordinated phosphorus atoms derivatives. ${ }^{1} \mathrm{H},{ }^{13} \mathrm{C},{ }^{31} \mathrm{P}$ NMR spectra were recorded with Bruker AC 200, AC 250, DPX 300 or AMX 400 spectrometers. References for NMR chemical shifts are 85\% $\mathrm{H}_{3} \mathrm{PO}_{4}$ for ${ }^{31} \mathrm{P} \mathrm{NMR}, \mathrm{SiMe}_{4}$ for ${ }^{1} \mathrm{H}$ and ${ }^{13} \mathrm{C}$ NMR. The attribution of ${ }^{13} \mathrm{C}$ NMR signals has been done using $J_{\text {mod }}$, two dimensional HMBC, and HMQC, Broad Band or $\mathrm{CW}^{31} \mathrm{P}$ decoupling experiments when necessary (bs means broad singlet). The following number scheme is used for NMR assignments.

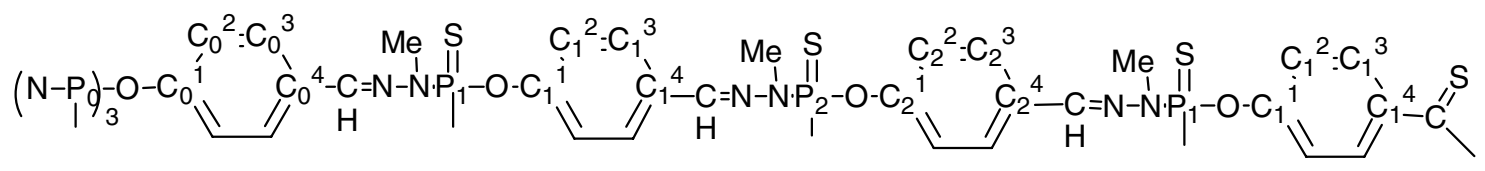

Dendrimers $\mathbf{1}-\mathbf{G}_{\mathbf{1}}$ and $\mathbf{1}-\mathbf{G}_{\mathbf{3}}$ were synthesized according to a published procedure:

Launay, N.; Caminade, A.M.; Majoral J.P. J. Organomet. Chem. 1997, 529, 51. 


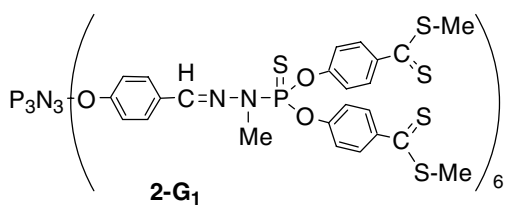

2-G $\mathbf{1}$ : A mixture of $\mathbf{1 - G _ { 1 }}(0.374 \mathrm{~g}, 0.2046 \mathrm{mmol}), \mathrm{HOC}_{6} \mathrm{H}_{4} \mathrm{C}(\mathrm{S}) \mathrm{SMe}(0.490 \mathrm{~g}, 2.663 \mathrm{mmol}, 7$ $\%$ excess) and $\mathrm{Cs}_{2} \mathrm{CO}_{3}(0.924 \mathrm{~g}, 2.836 \mathrm{mmol}, 15 \%$ excess $)$ in THF (30 mL) was stirred for 24 $\mathrm{h}$ at room temperature, then filtered. The solution was evaporated under vacuum, to afford a red powder, which was washed three times with ether $(3 \times 30 \mathrm{~mL})$. Compound $\mathbf{2}-\mathbf{G}_{\mathbf{1}}$ was isolated as a red powder in $95 \%$ yield $(0.700 \mathrm{~g})$.

${ }^{31} \mathrm{P}\left\{{ }^{1} \mathrm{H}\right\}$ NMR $\left(\mathrm{CDCl}_{3}, 101.2 \mathrm{MHz}\right): 11.6\left(\mathrm{~s}, \mathrm{P}_{0}\right), 64.1\left(\mathrm{~s}, \mathrm{P}_{1}\right) .{ }^{1} \mathrm{H} \mathrm{NMR}\left(\mathrm{CDCl}_{3}, 200.1 \mathrm{MHz}\right)$ : $2.71\left(\mathrm{~s}, 36 \mathrm{H}, \mathrm{SCH}_{3}\right), 3.25\left(\mathrm{~d},{ }^{3} J_{\mathrm{HP}}=10.6 \mathrm{~Hz}, 18 \mathrm{H}, \mathrm{NCH}_{3}\right), 6.99\left(\mathrm{~d},{ }^{3} J_{\mathrm{HH}}=8.7 \mathrm{~Hz}, 12 \mathrm{H}\right.$, Har), $7.17\left(\mathrm{~d},{ }^{3} J_{\mathrm{HH}}=9 \mathrm{~Hz}, 24 \mathrm{H}\right.$, Har), $7.55\left(\mathrm{~d},{ }^{3} J_{\mathrm{HH}}=8.7 \mathrm{~Hz}, 12 \mathrm{H}, \mathrm{Har}\right), 7.57$ (bs, $6 \mathrm{H}$, $\mathrm{CH}=\mathrm{N}), 7.94\left(\mathrm{~d},{ }^{3} J_{\mathrm{HH}}=8.7 \mathrm{~Hz}, 24 \mathrm{H}, \mathrm{Har}\right) .{ }^{13} \mathrm{C}\left\{{ }^{1} \mathrm{H}\right\} \mathrm{NMR}\left(\mathrm{CDCl}_{3}, 75.5 \mathrm{MHz}\right): 21.2(\mathrm{~s}$, $\left.\mathrm{SCH}_{3}\right), 33.4\left(\mathrm{~d},{ }^{2} J_{\mathrm{CP}}=12.6 \mathrm{~Hz}, \mathrm{NCH}_{3}\right), 121.5\left(\mathrm{~d},{ }^{3} J_{\mathrm{CP}}=4.8 \mathrm{~Hz}, \mathrm{C}_{1}{ }^{2}\right), 121.8\left(\mathrm{~s}, \mathrm{C}_{0}{ }^{2}\right), 128.7(\mathrm{~s}$, $\left.\mathrm{C}_{0}{ }^{3}\right), 128.8\left(\mathrm{~s}, \mathrm{C}_{1}^{3}\right), 132.3\left(\mathrm{~s}, \mathrm{C}_{0}{ }^{4}\right), 139.6\left(\mathrm{~d},{ }^{3} J_{\mathrm{CP}}=13.1 \mathrm{~Hz}, \mathrm{CH}=\mathrm{N}\right), 142.4\left(\mathrm{~s}, \mathrm{C}_{1}{ }^{4}\right), 152.7(\mathrm{~s}$, $\left.\mathrm{C}_{0}{ }^{1}\right), 154.2\left(\mathrm{~d},{ }^{2} J_{\mathrm{CP}}=7.2 \mathrm{~Hz}, \mathrm{C}_{1}{ }^{1}\right), 227.3(\mathrm{~s}, \mathrm{C}=\mathrm{S})$. Anal. Calcd for $\mathrm{C}_{144} \mathrm{H}_{132} \mathrm{~N}_{15} \mathrm{O}_{18} \mathrm{P}_{9} \mathrm{~S}_{30}$ (3601): C, 48.03; H, 3.69; N, 5.83. Found: C, 48.21; H, 3.72; N, 5.78.

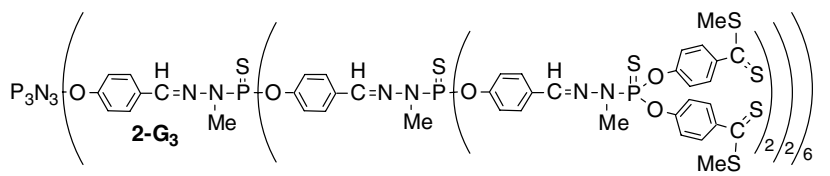

2-G $\mathbf{3}$ : A mixture of 1-G $\mathbf{G}_{\mathbf{3}}(0.100 \mathrm{~g}, 9.34 \mu \mathrm{mol}), \mathrm{HOC}_{6} \mathrm{H}_{4} \mathrm{C}(\mathrm{S}) \mathrm{SMe}(0.094 \mathrm{~g}, 0.5136 \mathrm{mmol}, 15$ $\%$ excess $)$ and $\mathrm{Cs}_{2} \mathrm{CO}_{3}(0.160 \mathrm{~g}, 0.4904 \mathrm{mmol}, 10 \%$ excess $)$ in THF (20 mL) was stirred for $36 \mathrm{~h}$ at room temperature, then centrifuged and filtered. The solution was evaporated under vacuum, to afford a red powder, which was washed three times with ether $(3 \times 30 \mathrm{~mL})$. Compound $\mathbf{2}-\mathbf{G}_{\mathbf{3}}$ was isolated as a red powder in $90 \%$ yield $(0.149 \mathrm{~g})$.

${ }^{31} \mathrm{P}\left\{{ }^{1} \mathrm{H}\right\}$ NMR $\left(\mathrm{CDCl}_{3}, 81.0 \mathrm{MHz}\right): 11.9$ (s, $\left.\mathrm{P}_{0}\right), 63.9$ (s, $\left.\mathrm{P}_{3}\right), 65.5\left(\mathrm{~s}, \mathrm{P}_{2}\right), 65.7$ (s, $\left.\mathrm{P}_{1}\right) .{ }^{1} \mathrm{H}$ NMR ( $\left.\mathrm{CDCl}_{3}, 200.1 \mathrm{MHz}\right): 2.64$ (s, $\left.144 \mathrm{H}, \mathrm{SCH}_{3}\right), 3.25$ (m, $\left.126 \mathrm{H}, \mathrm{NCH}_{3}\right)$, 6.8-7.2 (m, 186 $\mathrm{H}, \mathrm{Har}), 7.4-7.6(\mathrm{~m}, 120 \mathrm{H}, \mathrm{Har}, \mathrm{CH}=\mathrm{N}), 7.8-8.1$ (m, $96 \mathrm{H}, \mathrm{Har}) .{ }^{13} \mathrm{C}\left\{{ }^{1} \mathrm{H}\right\} \mathrm{NMR}\left(\mathrm{CDCl}_{3}\right.$, $50.3 \mathrm{MHz}$ ): $20.7\left(\mathrm{~s}, \mathrm{SCH}_{3}\right), 33.0\left(\mathrm{~d},{ }^{2} J_{\mathrm{CP}}=12.9 \mathrm{~Hz}, \mathrm{NCH}_{3}\right), 121.0\left(\mathrm{~d},{ }^{3} J_{\mathrm{CP}}=4.4 \mathrm{~Hz}, \mathrm{C}_{3}{ }^{2}\right)$, $121.8\left(\mathrm{bs}, \mathrm{C}_{0}{ }^{2}, \mathrm{C}_{1}{ }^{2}, \mathrm{C}_{2}{ }^{2}\right), 128.4\left(\mathrm{~s}, \mathrm{C}_{0}{ }^{3}, \mathrm{C}_{1}{ }^{3}, \mathrm{C}_{2}{ }^{3}, \mathrm{C}_{3}{ }^{3}\right), 131.9\left(\mathrm{bs}, \mathrm{C}_{0}^{4}, \mathrm{C}_{1}^{4}, \mathrm{C}_{2}^{4}\right), 139.3\left(\mathrm{~d},{ }^{3} J_{\mathrm{CP}}=\right.$ $13.4 \mathrm{~Hz}, \mathrm{CH}=\mathrm{N}), 141.9\left(\mathrm{~s}, \mathrm{C}_{3}{ }^{4}\right), 151.3\left(\mathrm{~s}, \mathrm{C}_{0}{ }^{1}, \mathrm{C}_{1}{ }^{1}, \mathrm{C}_{2}{ }^{1}\right), 153.8\left(\mathrm{~d},{ }^{2} J_{\mathrm{CP}}=7.1 \mathrm{~Hz}, \mathrm{C}_{3}{ }^{1}\right), 226.8$ (s, C=S). Anal. Calcd for $\mathrm{C}_{720} \mathrm{H}_{672} \mathrm{~N}_{87} \mathrm{O}_{90} \mathrm{P}_{45} \mathrm{~S}_{138}$ (17803): C, 48.58; H, 3.80; N, 6.85. Found: C, 48.71; H, 3.88; N, 6.78. 




3-G $\mathbf{G}_{\mathbf{1}}$ : Neat amino-azide 3 (0.045 g, $0.45 \mathrm{mmol}, 35 \%$ excess) was added onto a solution of 2$\mathbf{G}_{1}(0.100 \mathrm{~g}, 27.7 \mu \mathrm{mol})$ in THF $(3 \mathrm{~mL})$. The red solution was stirred for 24 hours at room temperature until the color change to deep yellow. The solvent was partially evaporated and the dendrimer was precipitated by addition of a large amount of pentane. The resulting yellow solid was washed several times with $\mathrm{Et}_{2} \mathrm{O}$, to afford $\mathbf{3}-\mathbf{G}_{\mathbf{1}}$ as a yellow powder in $86 \%$ yield $(0.100 \mathrm{~g})$.

${ }^{31} \mathrm{P}\left\{{ }^{1} \mathrm{H}\right\} \mathrm{NMR}\left(\mathrm{CDCl}_{3}, 81.0 \mathrm{MHz}\right): 12.1$ (s, $\left.\mathrm{P}_{0}\right), 64.9$ (s, $\left.\mathrm{P}_{1}\right) .{ }^{1} \mathrm{H}$ NMR $\left(\mathrm{CDCl}_{3}, 20.1 \mathrm{MHz}\right)$ : 1.92 ("quint", $\left.{ }^{3} J_{\mathrm{HH}}=6.3 \mathrm{~Hz}, 24 \mathrm{H}, \mathrm{CH}_{2} \mathrm{CH}_{2} \mathrm{CH}_{2}\right), 3.24\left(\mathrm{~d},{ }^{3} J_{\mathrm{HP}}=10.3 \mathrm{~Hz}, 18 \mathrm{H}, \mathrm{NCH}_{3}\right), 3.39$ $\left(\mathrm{t},{ }^{3} J_{\mathrm{HH}}=6.2 \mathrm{~Hz}, 24 \mathrm{H}, \mathrm{CH}_{2} \mathrm{~N}_{3}\right), 3.76\left(\mathrm{t},{ }^{3} J_{\mathrm{HH}}=6.6 \mathrm{~Hz}, 24 \mathrm{H}, \mathrm{CH}_{2} \mathrm{NH}\right), 6.91\left(\mathrm{~d},{ }^{3} J_{\mathrm{HH}}=7.9 \mathrm{~Hz}\right.$, $12 \mathrm{H}$, Har), $7.07\left(\mathrm{~d},{ }^{3} J_{\mathrm{HH}}=8.1 \mathrm{~Hz}, 24 \mathrm{H}, \mathrm{Har}\right), 7.52\left(\mathrm{~d},{ }^{3} J_{\mathrm{HH}}=8.1 \mathrm{~Hz}, 24 \mathrm{H}\right.$, Har), $7.61(\mathrm{~m}, 18$ $\mathrm{H}$, Har, $\mathrm{CH}=\mathrm{N}), 8.2$ (bs, $12 \mathrm{H}, \mathrm{NH}) .{ }^{13} \mathrm{C}\left\{{ }^{1} \mathrm{H}\right\} \mathrm{NMR}\left(\mathrm{CDCl}_{3}, 50.3 \mathrm{MHz}\right): 27.1\left(\mathrm{~s}, \mathrm{CH}_{2}\right), 33.1$ $\left(\mathrm{d},{ }^{2} J_{\mathrm{CP}}=12.7 \mathrm{~Hz}, \mathrm{NCH}_{3}\right), 44.6\left(\mathrm{~s}, \mathrm{CH}_{2} \mathrm{~N}_{3}\right), 49.7\left(\mathrm{~s}, \mathrm{CH}_{2} \mathrm{NH}\right), 121.1\left(\mathrm{~d},{ }^{3} J_{\mathrm{CP}}=4.1 \mathrm{~Hz}, \mathrm{C}_{1}{ }^{2}\right)$, $121.6\left(\mathrm{~s}, \mathrm{C}_{0}{ }^{2}\right), 128.3\left(\mathrm{~s}, \mathrm{C}_{0}{ }^{3}, \mathrm{C}_{1}{ }^{3}\right), 131.9\left(\mathrm{~s}, \mathrm{C}_{0}^{4}\right), 138.6\left(\mathrm{C}_{1}^{4}\right), 139.6\left(\mathrm{~d},{ }^{3} J_{\mathrm{CP}}=13.1 \mathrm{~Hz}\right.$, $\mathrm{N}=\mathrm{CH}), 151.3\left(\mathrm{~d},{ }^{2} J_{\mathrm{CP}}=4.9 \mathrm{~Hz}, \mathrm{C}_{0}{ }^{1}\right), 152.4\left(\mathrm{~d},{ }^{2} J_{\mathrm{CP}}=5.6 \mathrm{~Hz}, \mathrm{C}_{1}{ }^{1}\right), 197.6(\mathrm{C}=\mathrm{S}) . \mathrm{IR}(\mathrm{KBr})$ : $2095 \mathrm{~cm}^{-1}\left(v \mathrm{~N}_{3}\right.$ ) Anal. Calcd for $\mathrm{C}_{168} \mathrm{H}_{180} \mathrm{~N}_{63} \mathrm{O}_{18} \mathrm{P}_{9} \mathrm{~S}_{18}$ (4226): C, 47.75; H, 4.29; N, 20.88 . Found: C, 47.82; H, 4.35; N, 20.54.



4-G $\mathbf{G}_{\mathbf{1}}$ : Neat amino-alcohol 4 (0.015 g, $0.2455 \mathrm{mmol}, 47 \%$ excess $)$ was added onto a solution of $\mathbf{2}-\mathbf{G}_{\mathbf{1}}(0.050 \mathrm{~g}, 13.9 \mu \mathrm{mol})$ in THF $(2 \mathrm{~mL})$. The red solution was stirred for 12 hours at room temperature until the color change to deep yellow. The solvent was partially evaporated and the dendrimer was precipitated by addition of a large amount of pentane. The resulting yellow solid was washed few times with $\mathrm{Et}_{2} \mathrm{O}$ and $\mathrm{CH}_{2} \mathrm{Cl}_{2}$, to afford $\mathbf{4}-\mathbf{G}_{\mathbf{1}}$ as a yellow powder in $96 \%$ yield $(0.050 \mathrm{~g})$.

${ }^{31} \mathrm{P}\left\{{ }^{1} \mathrm{H}\right\}$ NMR $\left(\mathrm{CD}_{3} \mathrm{COCD}_{3}, 81.0 \mathrm{MHz}\right): 12.0\left(\mathrm{~s}, \mathrm{P}_{0}\right), 65.2\left(\mathrm{~s}, \mathrm{P}_{1}\right) .{ }^{1} \mathrm{H}$ NMR $\left(\mathrm{CD}_{3} \mathrm{COCD}_{3}\right.$, $200.1 \mathrm{MHz}): 3.37\left(\mathrm{~d},{ }^{3} J_{\mathrm{HP}}=10.6 \mathrm{~Hz}, 18 \mathrm{H}, \mathrm{NCH}_{3}\right), 3.87\left(\mathrm{~m}, 48 \mathrm{H}, \underline{\mathrm{C}}_{2}{ }^{\mathrm{NH}}, \mathrm{C}_{2} \underline{\mathrm{H}}\right), 4.3$ (bs, 
$12 \mathrm{H}, \mathrm{OH}), 7.09\left(\mathrm{~d},{ }^{3} J_{\mathrm{HH}}=8.3 \mathrm{~Hz}, 12 \mathrm{H}, \mathrm{Har}\right), 7.24\left(\mathrm{~d},{ }^{3} J_{\mathrm{HH}}=7.5 \mathrm{~Hz}, 24 \mathrm{H}, \mathrm{Har}\right), 7.68\left(\mathrm{~d},{ }^{3} J_{\mathrm{HH}}\right.$ $=8.5 \mathrm{~Hz}, 12 \mathrm{H}, \mathrm{Har}), 7.84\left(\mathrm{~d},{ }^{3} \mathrm{~J}_{\mathrm{HH}}=7.5 \mathrm{~Hz}, 30 \mathrm{H}, \mathrm{Har}, \mathrm{CH}=\mathrm{N}\right), 9.4$ (bs, $\left.12 \mathrm{H}, \mathrm{NH}\right) .{ }^{13} \mathrm{C}\left\{{ }^{1} \mathrm{H}\right\}$ NMR $\left(\mathrm{CD}_{3} \mathrm{COCD}_{3}, 62.9 \mathrm{MHz}\right): 33.5\left(\mathrm{~d},{ }^{2} J_{\mathrm{CP}}=13.0 \mathrm{~Hz}, \mathrm{CH}_{3} \mathrm{~N}\right), 49.8$ and $49.9\left(2 \mathrm{~s}, \mathrm{CH}_{2} \mathrm{NH}\right)$, $59.6\left(\mathrm{~s}, \mathrm{CH}_{2} \mathrm{OH}\right), 121.5\left(\mathrm{~d},{ }^{3} J_{\mathrm{CP}}=4.3 \mathrm{~Hz}, \mathrm{C}_{1}{ }^{2}\right), 122.0\left(\mathrm{~s}, \mathrm{C}_{0}{ }^{2}\right), 129.2\left(\mathrm{~s}, \mathrm{C}_{0}{ }^{3}\right), 129.6\left(\mathrm{~s}, \mathrm{C}_{1}{ }^{3}\right)$, $133.2\left(\mathrm{~s}, \mathrm{C}_{1}{ }^{4}\right), 139.7\left(\mathrm{~s}, \mathrm{C}_{0}{ }^{4}\right), 140.9\left(\mathrm{~d},{ }^{3} J_{\mathrm{CP}}=14.1 \mathrm{~Hz}, \mathrm{CH}=\mathrm{N}\right), 152.0\left(\mathrm{~s}, \mathrm{C}_{0}{ }^{1}\right), 153.1\left(\mathrm{~d},{ }^{2} J_{\mathrm{CP}}=\right.$ 7.6 Hz, $\mathrm{C}_{1}{ }^{1}$ ), 197.9 and 198.1 (2s, C=S). Anal. Calcd for $\mathrm{C}_{156} \mathrm{H}_{168} \mathrm{~N}_{27} \mathrm{O}_{30} \mathrm{P}_{9} \mathrm{~S}_{18}$ (3757): $\mathrm{C}$, 49.87; H, 4.51; N, 10.07. Found: C, 49.98; H, 4.57; N, 9.95.

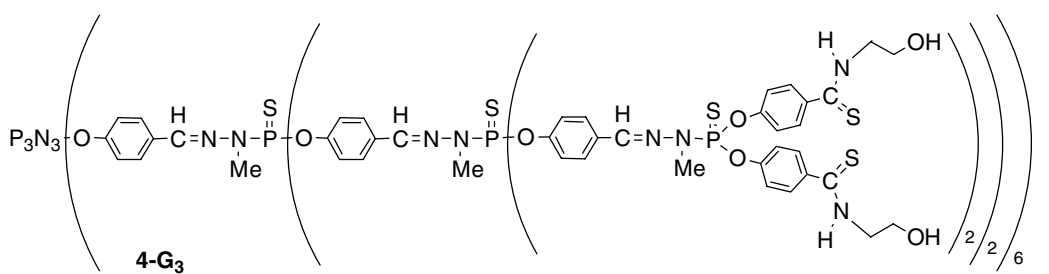

4-G3: Neat amino-alcohol $4(0.237 \mathrm{~g}, 3.88 \mathrm{mmol}, 40$ equiv/C=S $)$ was added onto a solution of 2-G $\mathbf{G}_{3}(0.036 \mathrm{~g}, 2.02 \mu \mathrm{mol})$ in $\mathrm{CHCl}_{3}(1 \mathrm{~mL})$. The red solution was stirred for $20 \mathrm{mn}$ at room temperature until the color change to deep yellow. The solvent was evaporated to dryness and the resulting yellow solid was washed 3 times with $\mathrm{Et}_{2} \mathrm{O}$, then 3 times with $\mathrm{EtOH}$, then with $\mathrm{Et}_{2} \mathrm{O}$ and pentane, to afford $\mathbf{4}-\mathbf{G}_{\mathbf{3}}$ as a yellow powder in $67 \%$ yield $(0.025 \mathrm{~g})$.

${ }^{31} \mathrm{P}\left\{{ }^{1} \mathrm{H}\right\}$ NMR $\left(\mathrm{CD}_{3} \mathrm{COCD}_{3}, 81.0 \mathrm{MHz}\right): 12.2\left(\mathrm{~s}, \mathrm{P}_{0}\right), 65.1\left(\mathrm{~s}, \mathrm{P}_{3}\right), 65.7\left(\mathrm{~s}, \mathrm{P}_{1}\right.$ and $\left.\mathrm{P}_{2}\right) .{ }^{1} \mathrm{H}$ NMR $\left(\mathrm{CD}_{3} \mathrm{COCD}_{3}, 200.1 \mathrm{MHz}\right): 3.34\left(\mathrm{bd},{ }^{3} J_{\mathrm{HP}}=10.2 \mathrm{~Hz}, 126 \mathrm{H}, \mathrm{NCH}_{3}\right), 3.85$ (bs, $192 \mathrm{H}$, $\mathrm{C}_{2} \mathrm{OH}, \underline{\mathrm{C}}_{2} \mathrm{NH}$ ), 4.2 (bs, $\left.48 \mathrm{H}, \mathrm{OH}\right), 7.01-7.20$ (m, $\left.180 \mathrm{H}, \mathrm{Har}\right), 7.60-7.90$ (m, $222 \mathrm{H}$, Har, $\mathrm{HC}=\mathrm{N}), 9.37$ (bs, $48 \mathrm{H}, \mathrm{NH}) .{ }^{13} \mathrm{C}\left\{{ }^{1} \mathrm{H}\right\} \mathrm{NMR}\left(\mathrm{CD}_{3} \mathrm{COCD}_{3}, 75.5 \mathrm{MHz}\right): 33.2\left(\mathrm{~m}, \mathrm{CH}_{3}\right), 49.6$ (s, $\left.\mathrm{CH}_{2} \mathrm{NH}\right), 59.2\left(\mathrm{~s}, \mathrm{CH}_{2} \mathrm{OH}\right), 121.1\left(\mathrm{~d},{ }^{3} J_{\mathrm{CP}}=4.5 \mathrm{~Hz}, \mathrm{C}_{3}{ }^{2}\right), 122.1\left(\mathrm{bs}, \mathrm{C}_{0}{ }^{2}, \mathrm{C}_{1}{ }^{2}, \mathrm{C}_{2}{ }^{2}\right), 128.8$ (bs, $\left.\mathrm{C}_{0}{ }^{3}, \mathrm{C}_{1}{ }^{3}, \mathrm{C}_{2}{ }^{3}\right), 129.3\left(\mathrm{~s}, \mathrm{C}_{3}{ }^{3}\right), 132.8\left(\mathrm{~m}, \mathrm{C}_{0}{ }^{4}, \mathrm{C}_{1}{ }^{4}, \mathrm{C}_{2}{ }^{4}\right), 139.2\left(\mathrm{~s}, \mathrm{C}_{3}{ }^{4}\right), 140.7(\mathrm{~m}, \mathrm{CH}=\mathrm{N})$, $151.9\left(\mathrm{~m}, \mathrm{C}_{0}{ }^{1}, \mathrm{C}_{1}{ }^{1}, \mathrm{C}_{2}{ }^{1}\right), 152.8\left(\mathrm{~d},{ }^{2} \mathrm{~J}_{\mathrm{CP}}=6.5 \mathrm{~Hz}, \mathrm{C}_{3}{ }^{1}\right), 197.5$ (s, C=S). Anal. Calcd for $\mathrm{C}_{768} \mathrm{H}_{816} \mathrm{~N}_{135} \mathrm{O}_{138} \mathrm{P}_{45} \mathrm{~S}_{90}$ (18425): C, 50.06; H, 4.46; N, 10.26. Found: C, 50.15; H, 4.49; N, 10.21 .

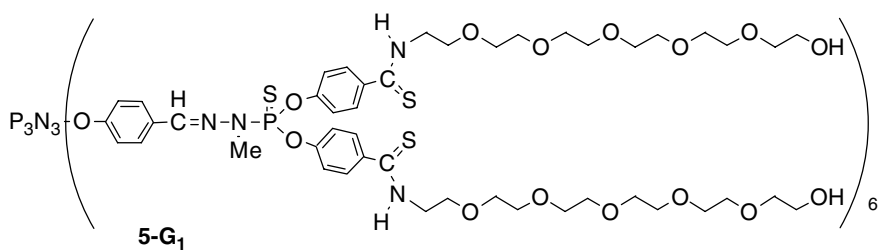

5-G $\mathbf{1}$ : Neat aminoglycol 5 (0.092 g, $0.327 \mathrm{mmol}, 60 \%$ excess $)$ was added onto a solution of 2$\mathbf{G}_{\mathbf{1}}(0.060 \mathrm{~g}, 16.7 \mu \mathrm{mol})$ in THF $(2 \mathrm{~mL})$. The red solution was stirred for 24 hours at room 
temperature until the color change to deep yellow. The solvent was evaporated to dryness and the resulting yellow solid was washed 3 times with $\mathrm{Et}_{2} \mathrm{O}$, then with pentane/THF, then with $\mathrm{Et}_{2} \mathrm{O}$, to afford $\mathbf{5}-\mathbf{G}_{\mathbf{1}}$ as a yellow waxy solid in $61 \%$ yield $(0.065 \mathrm{~g})$.

${ }^{31} \mathrm{P}\left\{{ }^{1} \mathrm{H}\right\}$ NMR $\left(\mathrm{CDCl}_{3}, 81.0 \mathrm{MHz}\right): 11.9\left(\mathrm{~s}, \mathrm{P}_{0}\right), 65.1$ (s, $\left.\mathrm{P}_{1}\right) .{ }^{1} \mathrm{H}$ NMR $\left(\mathrm{CDCl}_{3}, 200.1 \mathrm{MHz}\right)$ : $3.25\left(\mathrm{~d},{ }^{3} J_{\mathrm{HP}}=10.1 \mathrm{~Hz}, 18 \mathrm{H}, \mathrm{NCH}_{3}\right), 3.52\left(\mathrm{~m}, 240 \mathrm{H}, \mathrm{CH}_{2} \mathrm{O}\right), 3.75$ (bs, $\left.24 \mathrm{H}, \mathrm{C}_{2} \mathrm{OH}\right), 3.98$ (bs, $\left.36 \mathrm{H}, \mathrm{C}_{2} 2 \mathrm{NH}, \mathrm{OH}\right), 7.14$ (m, $\left.36 \mathrm{H}, \mathrm{Har}\right), 7.63$ (m, $\left.12 \mathrm{H}, \mathrm{Har}\right), 7.78$ (m, $30 \mathrm{H}$, Har, $\mathrm{CH}=\mathrm{N}), 9.34$ (bs, $12 \mathrm{H}, \mathrm{NH}) .{ }^{13} \mathrm{C}\left\{{ }^{1} \mathrm{H}\right\} \mathrm{NMR}\left(\mathrm{CDCl}_{3}, 62.9 \mathrm{MHz}\right): 33.1\left(\mathrm{~d}, \mathrm{CH}_{3} \mathrm{~N},{ }^{2} J_{\mathrm{CP}}=12.5\right.$ $\mathrm{Hz}$ ), 46.8 (s, $\left.\mathrm{CH}_{2} \mathrm{NH}\right), 61.3$ (s, $\left.\mathrm{CH}_{2} \mathrm{OH}\right), 68.2$ (s, $\mathrm{CH}_{2} \mathrm{CH}_{2} \mathrm{NH}_{2}$ ), 69.8 (s, $\underline{\mathrm{CH}}_{2} \mathrm{CH}_{2} \mathrm{O}$ ), 69.9 (s, $\mathrm{CH}_{2} \mathrm{O}$ ), 70.3 (s, $\left.\mathrm{CH}_{2} \mathrm{O}\right), 70.4$ (s, $\mathrm{CH}_{2} \mathrm{O}$ ), 70.5 (s, $\mathrm{CH}_{2} \mathrm{O}$ ), 72.6 (s, $\left.\underline{\mathrm{CH}}_{2} \mathrm{CH}_{2} \mathrm{OH}\right), 120.9$ (d, ${ }^{3} \mathrm{~J}_{\mathrm{CP}}$ $\left.=3.8 \mathrm{~Hz}, \mathrm{C}_{1}^{2}\right), 121.3\left(\mathrm{~s}, \mathrm{C}_{0}^{2}\right), 128.4\left(\mathrm{~s}, \mathrm{C}_{0}^{3}\right), 128.9\left(\mathrm{~s}, \mathrm{C}_{1}^{3}\right), 131.9\left(\mathrm{~s}, \mathrm{C}_{0}^{4}\right), 138.8\left(\mathrm{~s}, \mathrm{C}_{1}^{4}\right)$, $139.0\left(\mathrm{~d},{ }^{3} J_{\mathrm{CP}}=14.1 \mathrm{~Hz}, \mathrm{CH}=\mathrm{N}\right), 151.4\left(\mathrm{~s}, \mathrm{C}_{0}{ }^{1}\right), 152.4\left(\mathrm{~d},{ }^{2} J_{\mathrm{CP}}=4.7 \mathrm{~Hz}, \mathrm{C}_{1}{ }^{1}\right), 197.3(\mathrm{~s}, \mathrm{C}=\mathrm{S})$. Anal. Calcd for $\mathrm{C}_{276} \mathrm{H}_{408} \mathrm{~N}_{27} \mathrm{O}_{90} \mathrm{P}_{9} \mathrm{~S}_{18}$ (6400): C, 51.80; H, 6.43; N, 5.91. Found: C, 51.73; H, $6.48 ; \mathrm{N}, 5.87$.

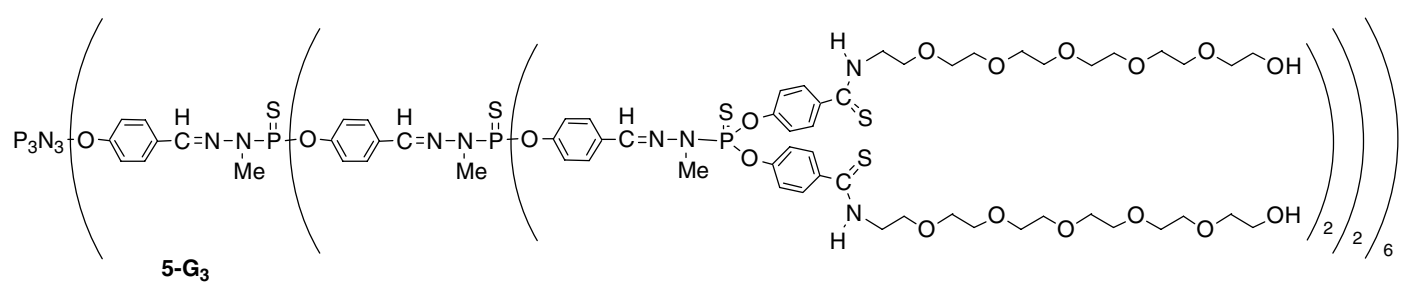

5-G : Neat aminoglycol 5 (0.048 g, $0.170 \mathrm{mmol}, 26 \%$ excess) was added onto a solution of 2$\mathbf{G}_{3}(0.050 \mathrm{~g}, 2.8 \mu \mathrm{mol})$ in $\mathrm{CHCl}_{3}(2 \mathrm{~mL})$. The red solution was stirred for 48 hours at room temperature, 4 days at $50^{\circ} \mathrm{C}$ until the color change to deep yellow. The solvent was evaporated to dryness and the resulting yellow solid was washed with $\mathrm{CH}_{3} \mathrm{CN}, \mathrm{THF}, \mathrm{Et}_{2} \mathrm{O}$, then with pentane, to afford $\mathbf{5}-\mathbf{G}_{3}$ as a yellow waxy solid in $50 \%$ yield $(0.041 \mathrm{~g})$.

${ }^{31} \mathrm{P}\left\{{ }^{1} \mathrm{H}\right\}$ NMR $\left(\mathrm{CDCl}_{3}, 81.0 \mathrm{MHz}\right): 11.7\left(\mathrm{~s}, \mathrm{P}_{0}\right), 64.8\left(\mathrm{~s}, \mathrm{P}_{3}\right), 65.8\left(\mathrm{~s}, \mathrm{P}_{2}\right), 66.0\left(\mathrm{~s}, \mathrm{P}_{1}\right) .{ }^{1} \mathrm{H}$ NMR $\left(\mathrm{CDCl}_{3}, 200.1 \mathrm{MHz}\right): 3.15$ (m, $\left.126 \mathrm{H}, \mathrm{NCH}_{3}\right), 3.51-3.75\left(\mathrm{~m}, 1056 \mathrm{H}, \underline{\mathrm{C}}_{2} \mathrm{O}\right), 3.97$ (bs, $144 \mathrm{H}$, $\mathrm{NCH}_{2}, \mathrm{OH}$ ), 7.17 (m, $\left.186 \mathrm{H}, \mathrm{Har}\right), 7.63$ (m, 120 H, Har, HC=N), 7.80 (m, 96 H, Har), 9.25 (bs, $48 \mathrm{H}, \mathrm{NH}) .{ }^{13} \mathrm{C}\left\{{ }^{1} \mathrm{H}\right\} \mathrm{NMR}\left(\mathrm{CDCl}_{3}, 50.3 \mathrm{MHz}, \delta \mathrm{ppm}\right): 33.0\left(\mathrm{~d},{ }^{2} J_{\mathrm{CP}}=12.6 \mathrm{~Hz}, \mathrm{NCH}_{3}\right)$, 46.6 (s, $\left.\mathrm{CH}_{2} \mathrm{NH}\right), 61.3$ (s, $\left.\mathrm{CH}_{2} \mathrm{OH}\right), 68.1$ (s, $\left.\mathrm{CH}_{2} \mathrm{CH}_{2} \mathrm{NH}\right), 69.9$ (s, $\left.\mathrm{CH}_{2} \mathrm{OH}\right), 70.3$ (s, $\left.\mathrm{CH}_{2} \mathrm{O}\right)$, 72.5 (s, $\mathrm{CH}_{2} \mathrm{O}$ ), 121.0 (bs, $\mathrm{C}_{3}^{2}$ ), $121.8\left(\mathrm{~m}, \mathrm{C}_{0}^{2}, \mathrm{C}_{1}^{2}, \mathrm{C}_{2}^{2}\right), 128.4\left(\mathrm{bs}, \mathrm{C}_{0}^{3}, \mathrm{C}_{1}^{3}, \mathrm{C}_{2}^{3}\right), 128.9\left(\mathrm{~s}, \mathrm{C}_{3}^{3}\right)$, $132.1\left(\mathrm{~m}, \mathrm{C}_{0}{ }^{4}, \mathrm{C}_{1}{ }^{4}, \mathrm{C}_{2}{ }^{4}\right), 138.7\left(\mathrm{~s}, \mathrm{C}_{3}{ }^{4}\right), 139.5(\mathrm{~m}, \mathrm{CH}=\mathrm{N}), 151.6\left(\mathrm{~m}_{1} \mathrm{C}_{0}{ }^{1}, \mathrm{C}_{1}{ }^{1}, \mathrm{C}_{2}{ }^{1}\right), 152.4(\mathrm{~d}$, $\left.{ }^{2} J_{\mathrm{CP}}=7.3 \mathrm{~Hz}, \mathrm{C}_{3}{ }^{1}\right), 195.4(\mathrm{~s}, \mathrm{C}=\mathrm{S})$. Anal. Calcd for $\mathrm{C}_{1248} \mathrm{H}_{1776} \mathrm{~N}_{135} \mathrm{O}_{378} \mathrm{P}_{45} \mathrm{~S}_{90}(28998): \mathrm{C}, 51.69 ; \mathrm{H}$, 6.17; N, 6.52. Found: C, 51.73; H, 6.20; N, 6.48. 


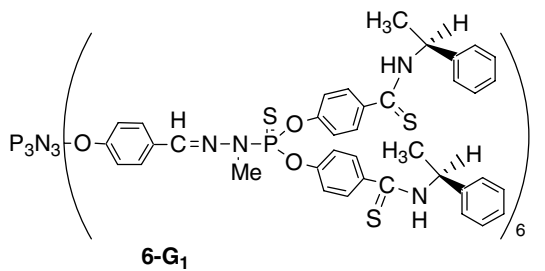

6-G $\mathbf{G}_{1}$ : Neat methylbenzylamine $6(30 \mu \mathrm{L}, 0.233 \mathrm{mmol}, 20 \%$ excess $)$ was added onto a solution of $\mathbf{2}-\mathbf{G}_{\mathbf{1}}(0.055 \mathrm{~g}, 16 \mu \mathrm{mol})$ in THF $(2 \mathrm{~mL})$. The red solution was stirred for 4 hours at room temperature until the color change to deep yellow. The solvent was evaporated to dryness and the resulting yellow solid was washed 2 times with $\mathrm{Et}_{2} \mathrm{O}$, then with pentane/THF, to afford $\mathbf{6}-\mathbf{G}_{\mathbf{1}}$ as a yellow solid in $98 \%$ yield $(0.070 \mathrm{~g})$.

${ }^{31} \mathrm{P}\left\{{ }^{1} \mathrm{H}\right\}$ NMR $\left(\mathrm{CDCl}_{3}, 81.0 \mathrm{MHz}\right): 12.3$ (s, $\left.\mathrm{P}_{0}\right), 65.1$ (s, $\left.\mathrm{P}_{1}\right) .{ }^{1} \mathrm{H}$ NMR $\left(\mathrm{CDCl}_{3}, 300.1 \mathrm{MHz}\right)$ : $1.65\left(\mathrm{~d},{ }^{3} J_{\mathrm{HH}}=6.6 \mathrm{~Hz}, 36 \mathrm{H}, \mathrm{CH}_{3} \mathrm{CH}\right), 3.15\left(\mathrm{~d},{ }^{3} J_{\mathrm{HP}}=10.2 \mathrm{~Hz}, 18 \mathrm{H}, \mathrm{NCH}_{3}\right), 5.81\left(\mathrm{q},{ }^{3} J_{\mathrm{HH}}=\right.$ $7.5 \mathrm{~Hz}, 12 \mathrm{H}, \mathrm{C} \underline{\mathrm{HNH}}), 6.92\left(\mathrm{~d},{ }^{3} J_{\mathrm{HH}}=8.4 \mathrm{~Hz}, 12 \mathrm{H}, \mathrm{Har}\right), 7.05\left(\mathrm{~d},{ }^{3} J_{\mathrm{HH}}=8.1 \mathrm{~Hz}, 24 \mathrm{H}, \mathrm{Har}\right)$, $7.30(\mathrm{~m}, 48 \mathrm{H}, \mathrm{Har}), 7.50\left(\mathrm{~d},{ }^{3} J_{\mathrm{HH}}=8.4 \mathrm{~Hz}, 24 \mathrm{H}, \mathrm{Har}\right), 7.59\left(\mathrm{~d},{ }^{3} J_{\mathrm{HH}}=9.0 \mathrm{~Hz}, 18 \mathrm{H}\right.$, Har, $\mathrm{CH}=\mathrm{N}), 7.84\left(\mathrm{~d},{ }^{3} \mathrm{~J}_{\mathrm{HH}}=7.6 \mathrm{~Hz}, 6 \mathrm{H}, \mathrm{Har}\right), 7.94\left(\mathrm{~d},{ }^{3} \mathrm{~J}_{\mathrm{HH}}=7.5 \mathrm{~Hz}, 6 \mathrm{H}, \mathrm{Har}\right) .{ }^{13} \mathrm{C}\left\{{ }^{1} \mathrm{H}\right\} \mathrm{NMR}$ $\left(\mathrm{CDCl}_{3}, 75.5 \mathrm{MHz}\right): 20.6$ and $20.8\left(2 \mathrm{~s}, \mathrm{CH}_{3}\right), 33.5\left(\mathrm{~d},{ }^{2} J_{\mathrm{CP}}=12.1 \mathrm{~Hz}, \mathrm{NCH}_{3}\right), 55.8(2 \mathrm{~s}$, $\mathrm{CHNH}), 121.5\left(\mathrm{~d},{ }^{2} J_{\mathrm{CP}}=4.9 \mathrm{~Hz}, \mathrm{C}_{1}^{2}\right), 121.6\left(\mathrm{~d},{ }^{2} J_{\mathrm{CP}}=4.8 \mathrm{~Hz}, \mathrm{C}_{1}^{2}\right), 122.1\left(\mathrm{~m}, \mathrm{C}_{0}^{2}\right), 127.0(\mathrm{~s}$, 2 Car), $128.3\left(\mathrm{~s}, \mathrm{C}_{0}{ }^{3}\right), 128.8\left(2 \mathrm{~s}, \mathrm{C}_{1}^{3}\right), 129.2$ (2s, 2 Car), $132.3\left(\mathrm{~s}, \mathrm{C}_{0}^{4}\right), 139.4$ (s, Car), 139.9 $\left(\mathrm{d},{ }^{3} J_{\mathrm{CP}}=13.3 \mathrm{~Hz}, \mathrm{CH}=\mathrm{N}\right), 141.6$ and $141.7\left(2 \mathrm{~s}, \mathrm{C}_{1}{ }^{4}\right), 151.7\left(\mathrm{~m}, \mathrm{C}_{0}{ }^{1}\right), 152.7\left(2 \mathrm{~d},{ }^{2} J_{\mathrm{CP}}=6.3\right.$ and 6.0 Hz, $\mathrm{C}_{1}{ }^{1}$ ), 196.8 (2s, C=S). Anal. Calcd for $\mathrm{C}_{228} \mathrm{H}_{216} \mathrm{~N}_{27} \mathrm{O}_{18} \mathrm{P}_{9} \mathrm{~S}_{18}$ (4478): C, 61.15; $\mathrm{H}$, 4.86; N, 8.45. Found: C, 61.27; H, 4.91; N, 8.39.

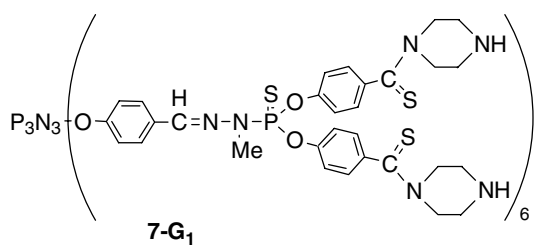

7-G $\mathbf{1}$ : Dendrimer 2-G $\mathbf{1}(0.098 \mathrm{~g}, 27.2 \mu \mathrm{mol})$ in THF $(45 \mathrm{~mL})$ was added over 5 hours to a solution of piperazine $7(3.13 \mathrm{~g}, 111$ equiv/C=S $)$ in THF $(20 \mathrm{~mL})$. After addition, the solution was stirred 1.5 hour, the solvent was removed under reduced pressure and the residual solid was washed several times with $\mathrm{Et}_{2} \mathrm{O}$. The powder was recovered and precipitated twice in

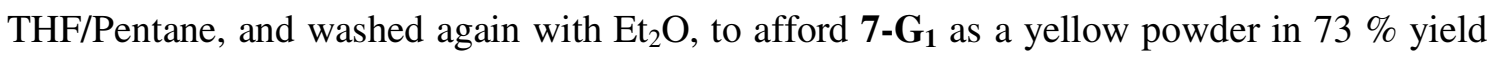
(0.081 g).

${ }^{31} \mathrm{P}\left\{{ }^{1} \mathrm{H}\right\}$ NMR $\left(\mathrm{CDCl}_{3}, 101.2 \mathrm{MHz}\right): 11.5\left(\mathrm{~s}, \mathrm{P}_{0}\right), 64.6\left(\mathrm{~s}, \mathrm{P}_{1}\right) .{ }^{1} \mathrm{H} \mathrm{NMR}\left(\mathrm{CDCl}_{3}, 250.1 \mathrm{MHz}\right)$ : 2.43 (bs, $\left.24 \mathrm{H}, \underline{\mathrm{C}}_{2} \mathrm{NH}\right), 2.92$ (bs, $24 \mathrm{H}, \mathrm{C}_{2}{ }_{2} \mathrm{NH}$ ), 3.29 (d, ${ }^{3} J_{\mathrm{HP}}=10.4 \mathrm{~Hz}, 18 \mathrm{H}, \mathrm{NCH}_{3}$ ), 3.48 
(bs, $24 \mathrm{H}, \mathrm{CH}_{2} \mathrm{NCS}$ ), 4.33 (bs, $24 \mathrm{H}, \mathrm{CH}_{2} \mathrm{NCS}$ ), 7.02 (d, ${ }^{3} J_{\mathrm{HH}}=8.5 \mathrm{~Hz}, 12 \mathrm{H}, \mathrm{Har}$ ), 7.19 (m, $36 \mathrm{H}, \mathrm{Har}), 7.58(\mathrm{~m}, 30 \mathrm{H}, \mathrm{Har}, \mathrm{CH}=\mathrm{N}) \cdot{ }^{13} \mathrm{C}\left\{{ }^{1} \mathrm{H}\right\} \mathrm{NMR}\left(\mathrm{CDCl}_{3}, 50.3 \mathrm{MHz}\right): 33.1\left(\mathrm{~d},{ }^{2} J_{\mathrm{CP}}=\right.$ 12.2 Hz, $\mathrm{CH}=\mathrm{N}), 45.6\left(\mathrm{~s}, \mathrm{CH}_{2} \mathrm{NH}\right), 46.5\left(\mathrm{~s}, \mathrm{CH}_{2} \mathrm{NH}\right), 50.7\left(\mathrm{~s}, \mathrm{CH}_{2} \mathrm{~N}\right), 53.5\left(\mathrm{~s}, \mathrm{CH}_{2} \mathrm{~N}\right), 121.2$ $\left(\mathrm{s}, \mathrm{C}_{0}{ }^{2}\right), 121.3\left(\mathrm{~s}, \mathrm{C}_{1}{ }^{2}\right), 127.4\left(\mathrm{~s}, \mathrm{C}_{1}{ }^{3}\right), 128.3\left(\mathrm{~s}, \mathrm{C}_{0}{ }^{3}\right), 131.9\left(\mathrm{~s}, \mathrm{C}_{0}{ }^{4}\right), 139.2\left(\mathrm{~d},{ }^{3} J_{\mathrm{CP}}=14.2 \mathrm{~Hz}\right.$, $\mathrm{CH}=\mathrm{N}), 139.9\left(\mathrm{~s}, \mathrm{C}_{1}{ }^{4}\right), 150.5\left(\mathrm{~d},{ }^{2} J_{\mathrm{CP}}=6.9 \mathrm{~Hz}, \mathrm{C}_{1}{ }^{1}\right), 151.2\left(\mathrm{~s}, \mathrm{C}_{0}{ }^{1}\right), 198.9(\mathrm{~s}, \mathrm{C}=\mathrm{S})$. Anal. Calcd for $\mathrm{C}_{180} \mathrm{H}_{204} \mathrm{~N}_{39} \mathrm{O}_{18} \mathrm{P}_{9} \mathrm{~S}_{18}$ (4058): C, 53.28; H, 5.07; N, 13.46. Found: C, 53.41; H, 5.13; N, 13.33 .

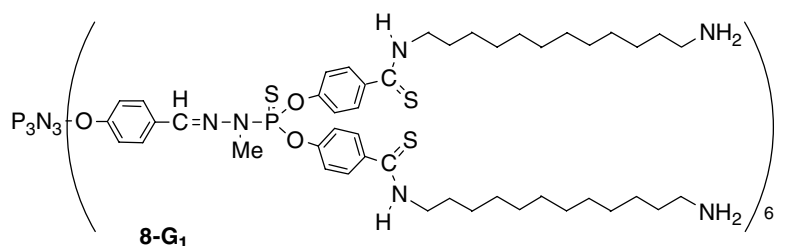

8-G $\mathbf{G}_{\mathbf{1}}$ : Dendrimer $\mathbf{2}-\mathbf{G}_{\mathbf{1}}(0.140 \mathrm{~g}, 38.9 \mu \mathrm{mol})$ in THF $(30 \mathrm{~mL})$ was added over 5 hours to a solution of diamine $8(6.0 \mathrm{~g}, 64$ equiv/C=S) in THF $(10 \mathrm{~mL})$. After addition, the solution was stirred 1.5 hour, the solvent was removed under reduced pressure and the residual solid was washed with hot $\mathrm{CH}_{3} \mathrm{CN}$. Evaporation to dryness of this solution allowed recovering $5.67 \mathrm{~g}$ of pure diamine 8 (96\%). The powder which was previously washed with acetonitrile was washed with $\mathrm{EtOH}$, then with $\mathrm{THF} /$ pentane, to afford $\mathbf{8}-\mathbf{G}_{\mathbf{1}}$ as a yellow powder in $47 \%$ yield $(0.099 \mathrm{~g})$.

${ }^{31} \mathrm{P}\left\{{ }^{1} \mathrm{H}\right\} \mathrm{NMR}\left(\mathrm{CDCl}_{3} / \mathrm{CD}_{3} \mathrm{OD}, 101.2 \mathrm{MHz}\right): 12.2$ (s), 65.1 (s). ${ }^{1} \mathrm{H} \mathrm{NMR}\left(\mathrm{CDCl}_{3} / \mathrm{CD}_{3} \mathrm{OD}\right.$, $250.1 \mathrm{MHz}$ ): 1.25 (bs, $192 \mathrm{H}, \mathrm{CH}_{2}$ ), 1.71 (bs, $48 \mathrm{H}, \mathrm{NH}_{2} \mathrm{CH}_{2} \underline{\mathrm{C}}_{2}$ ), 2.58 (q, ${ }^{3} J_{\mathrm{HH}}=6.3 \mathrm{~Hz}, 24$ $\mathrm{H}, \mathrm{H}_{2} \mathrm{NC}_{2}$ ), 3.23 (d, ${ }^{3} J_{\mathrm{HP}}=7.9 \mathrm{~Hz}, 18 \mathrm{H}, \mathrm{NCH}_{3}$ ), 3.68 (m, $\left.24 \mathrm{H}, \mathrm{C}_{2}{ }_{2} \mathrm{NHCS}\right), 6.94-7.10$ (m, $36 \mathrm{H}, \mathrm{Har}), 7.54(\mathrm{~m}, 42 \mathrm{H}, \mathrm{Har}, \mathrm{CH}=\mathrm{N}), 8.4(\mathrm{bs}, 12 \mathrm{H}, \mathrm{NH}) .{ }^{13} \mathrm{C}\left\{{ }^{1} \mathrm{H}\right\} \mathrm{NMR}\left(\mathrm{CDCl}_{3} /\right.$ $\mathrm{CD}_{3} \mathrm{OD}, 75.5 \mathrm{MHz}$ ): 26.5 (s, $\left.\mathrm{CH}_{2}\right), 26.7$ (s, $\left.\mathrm{CH}_{2}\right), 27$ (s, $\left.\mathrm{CH}_{2}\right), 28.9\left(\mathrm{~s}, \mathrm{CH}_{2}\right), 29\left(\mathrm{~s}, \mathrm{CH}_{2}\right), 29.2$ (s, $\left.\mathrm{CH}_{2}\right), 31.9\left(\mathrm{~m}, \mathrm{NCH}_{3}\right), 41\left(\mathrm{~s}, \mathrm{CH}_{2} \mathrm{NH}_{2}\right), 46.4\left(\mathrm{~s}, \mathrm{CH}_{2} \mathrm{NH}\right), 120.5\left(\mathrm{~s}, \mathrm{C}_{1}^{2}\right), 121\left(\mathrm{~s}, \mathrm{C}_{0}^{2}\right), 128$ $\left(\mathrm{s}, \mathrm{C}_{0}{ }^{3}\right), 128.3\left(\mathrm{~s}, \mathrm{C}_{1}{ }^{3}\right), 131.8\left(\mathrm{~d},{ }^{2} J_{\mathrm{CP}}=4.8 \mathrm{~Hz}, \mathrm{C}_{0}{ }^{4}\right), 138.8\left(\mathrm{~s}, \mathrm{C}_{1}{ }^{4}\right), 139.2\left(\mathrm{~d},{ }^{3} J_{\mathrm{CP}}=10.1 \mathrm{~Hz}\right.$, $\mathrm{CH}=\mathrm{N}), 150.9\left(\mathrm{~s}, \mathrm{C}_{0}^{1}\right), 151.9\left(\mathrm{~d},{ }^{2} J_{\mathrm{CP}}=7.1 \mathrm{~Hz}, \mathrm{C}_{1}{ }^{1}\right), 196.8(\mathrm{~s}, \mathrm{C}=\mathrm{S})$. Anal. Calcd for $\mathrm{C}_{276} \mathrm{H}_{420} \mathrm{~N}_{39} \mathrm{O}_{18} \mathrm{P}_{9} \mathrm{~S}_{18}$ (5429): C, 61.07; H, 7.80; N, 10.06. Found: C, 61.14; H, 7.87; N, 9.98. 

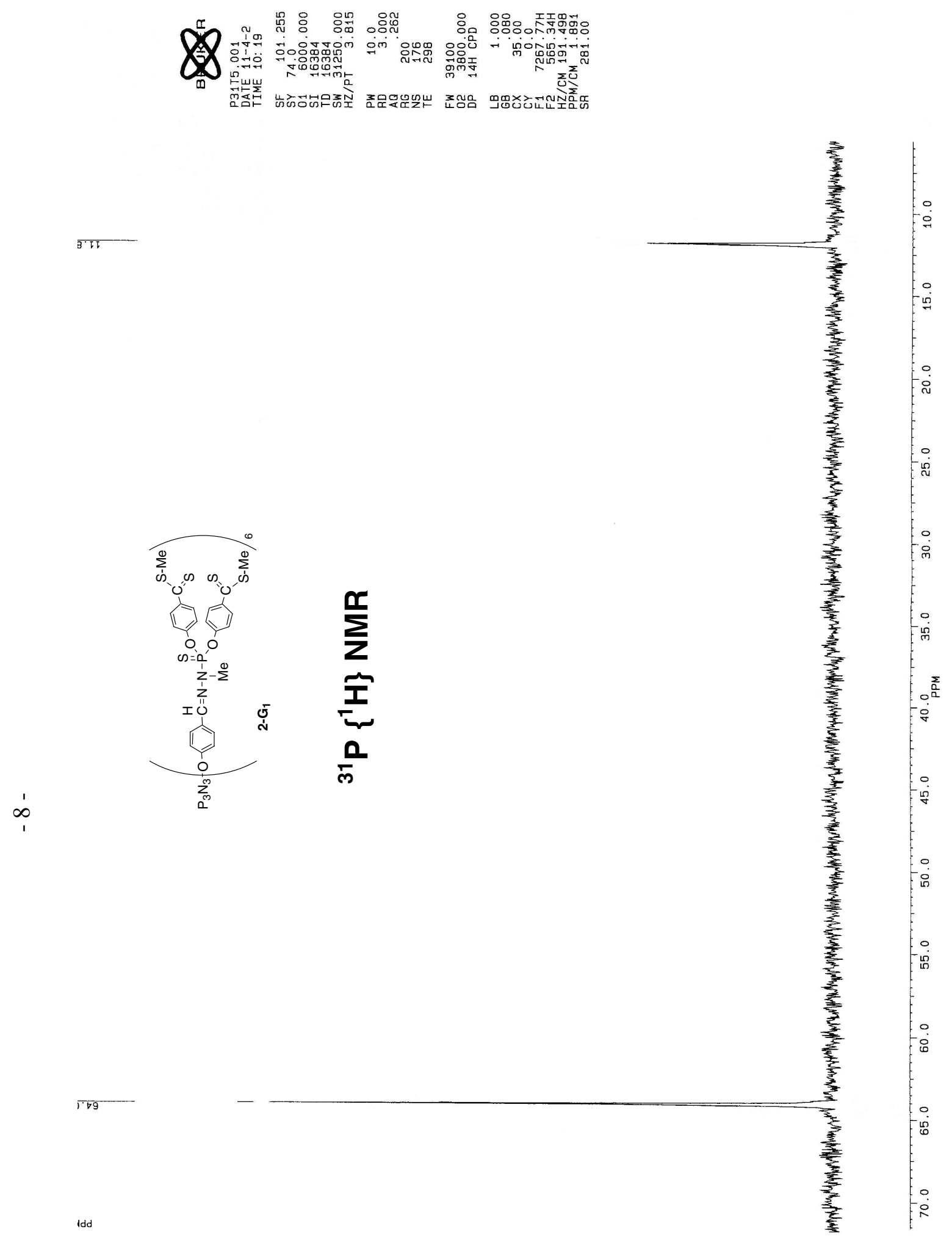


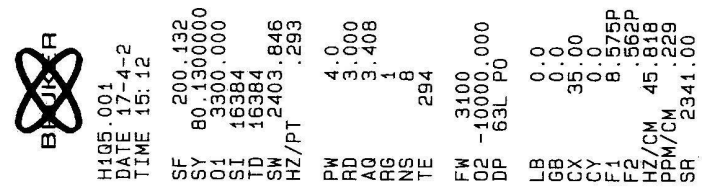

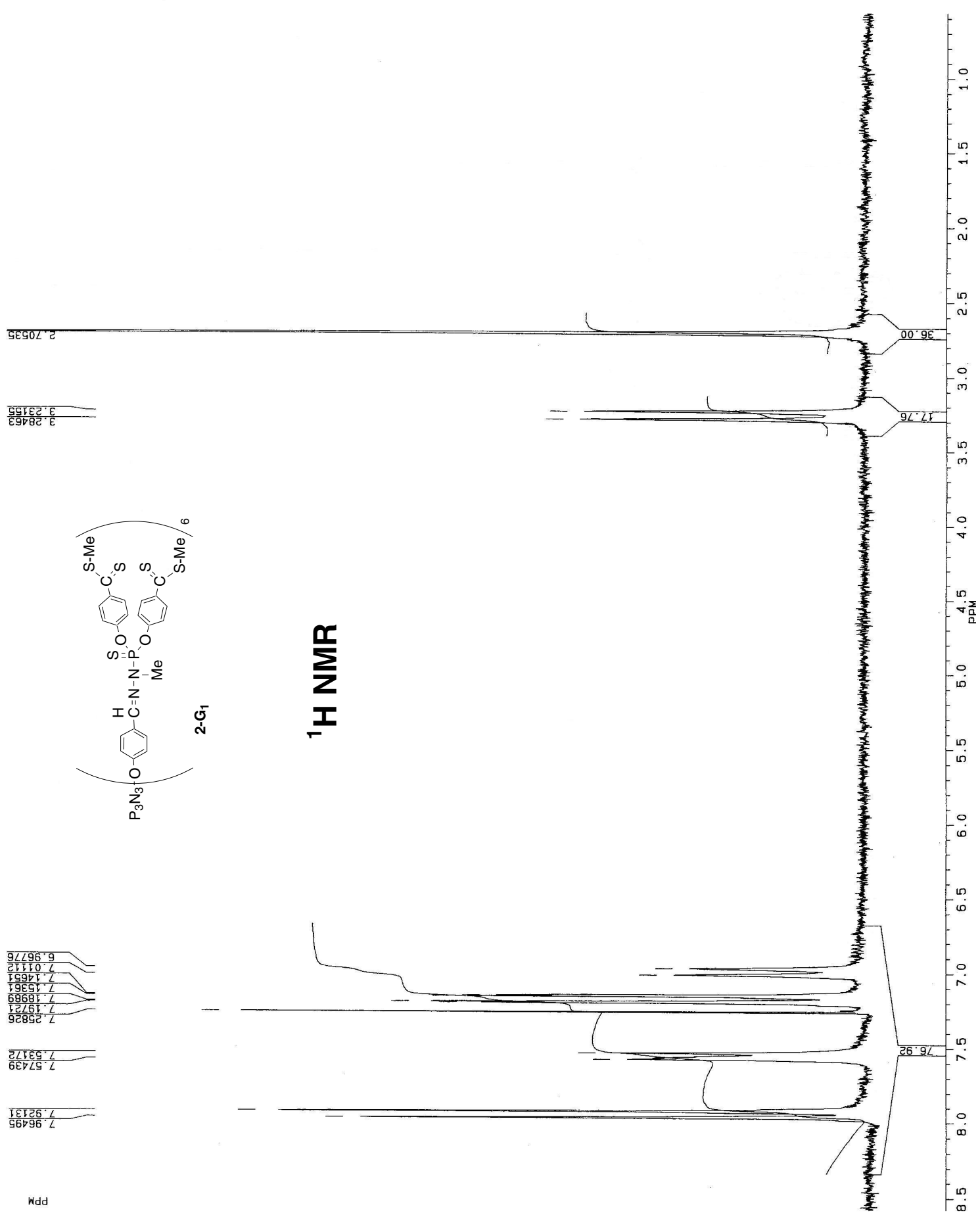




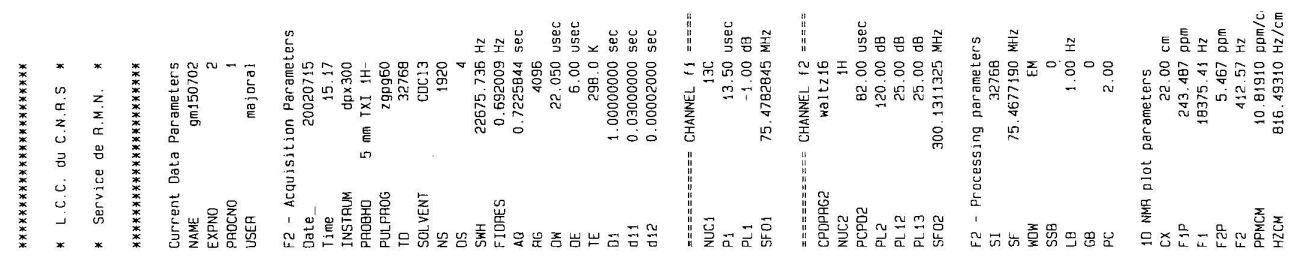

ट 记-

$\varepsilon \cdot \varepsilon E \longrightarrow$

$1 \cdot L L \longrightarrow$
$5 \cdot L$
$6 \cdot L L$

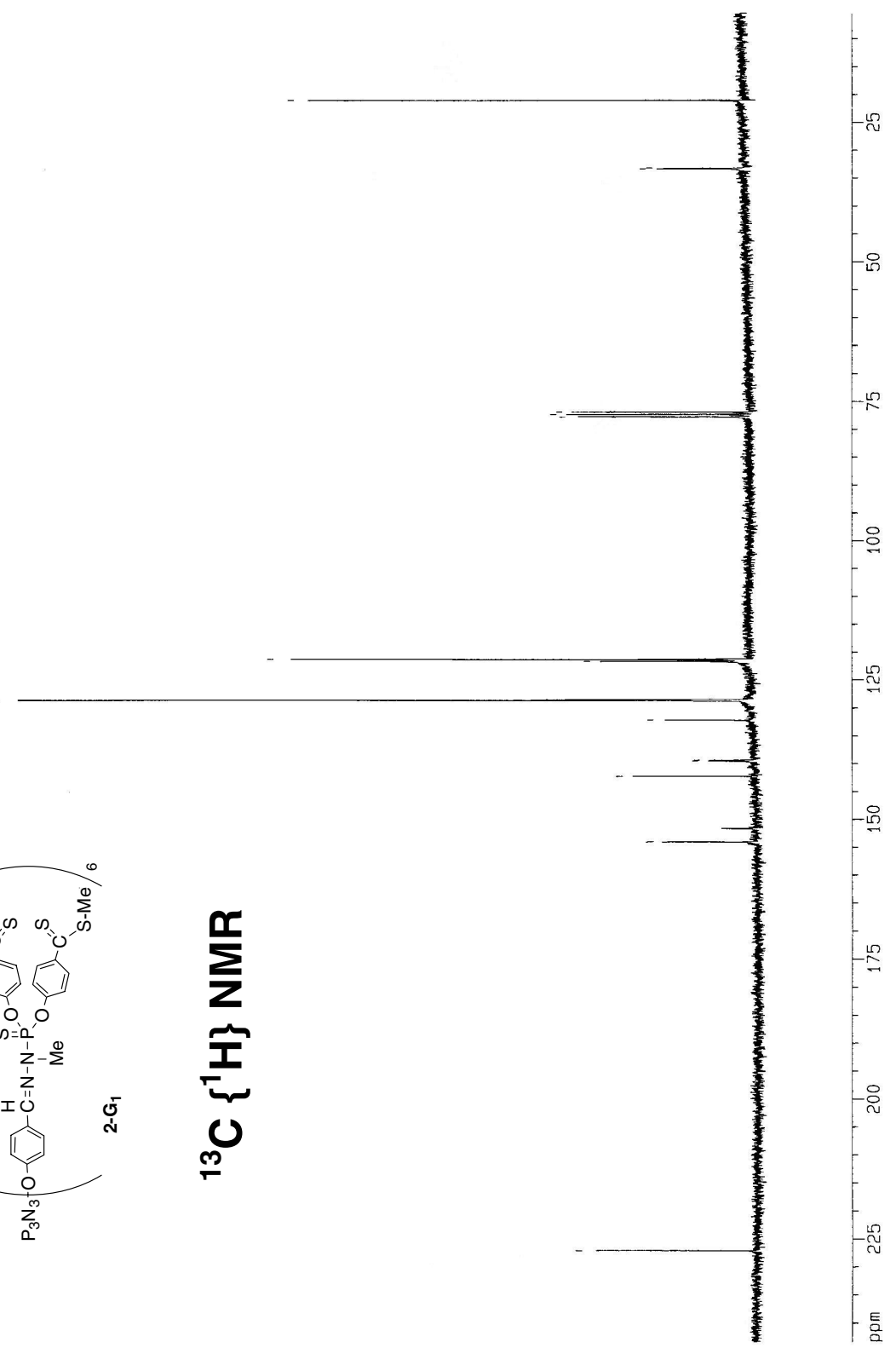



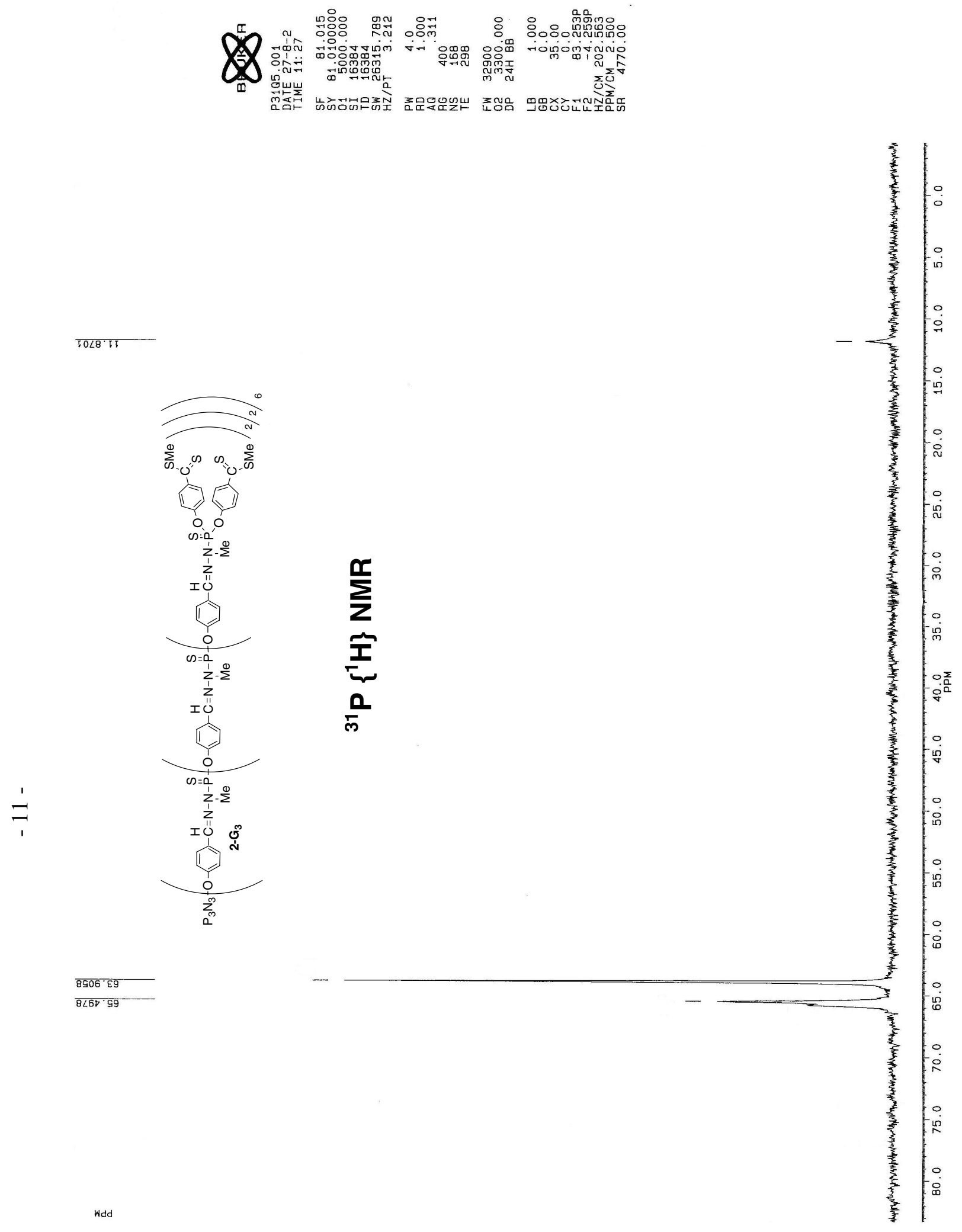


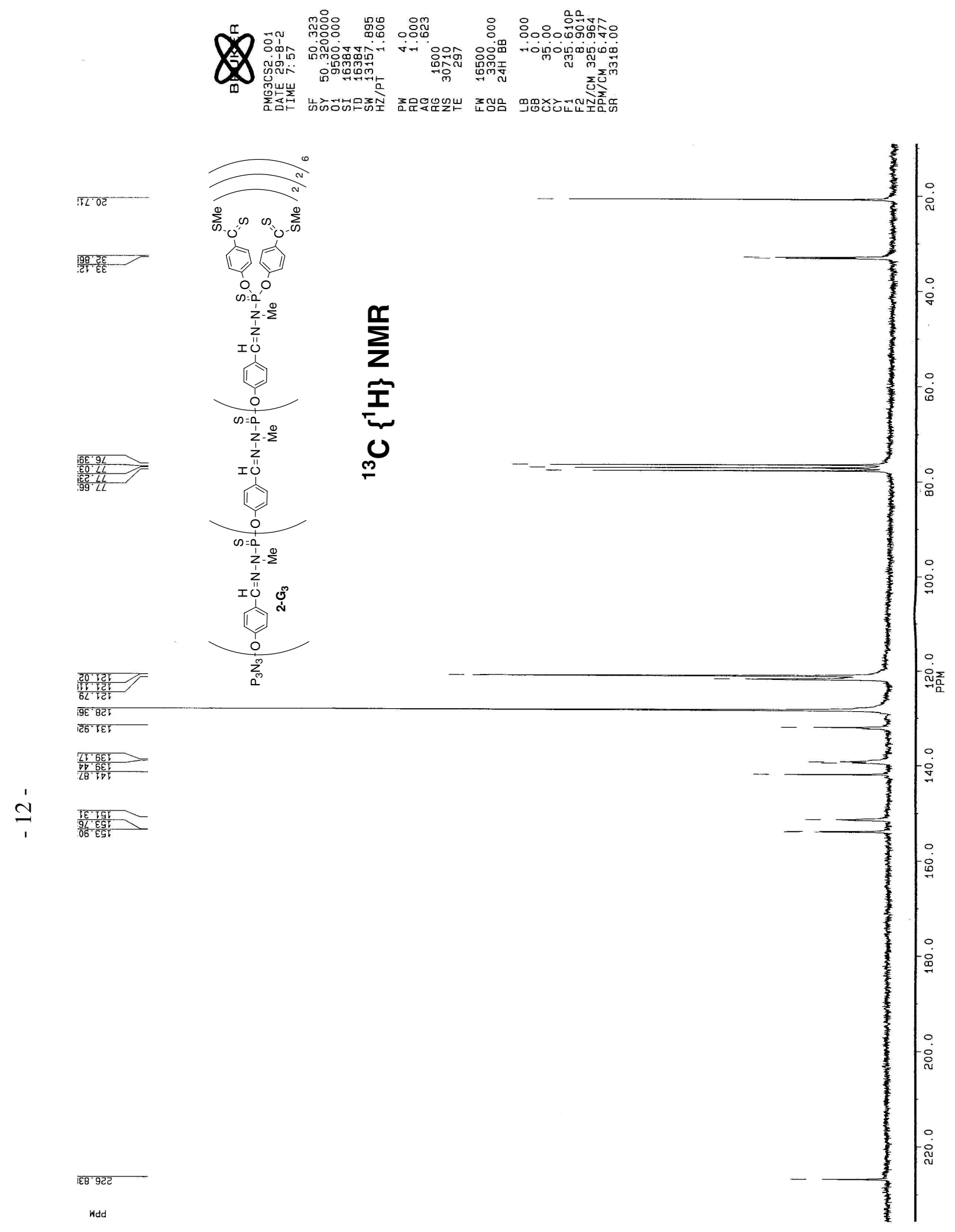






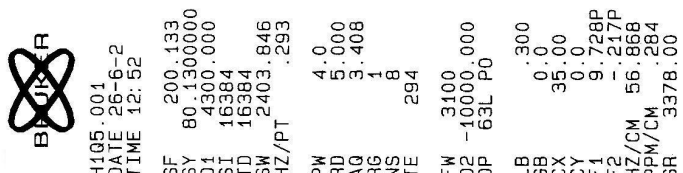

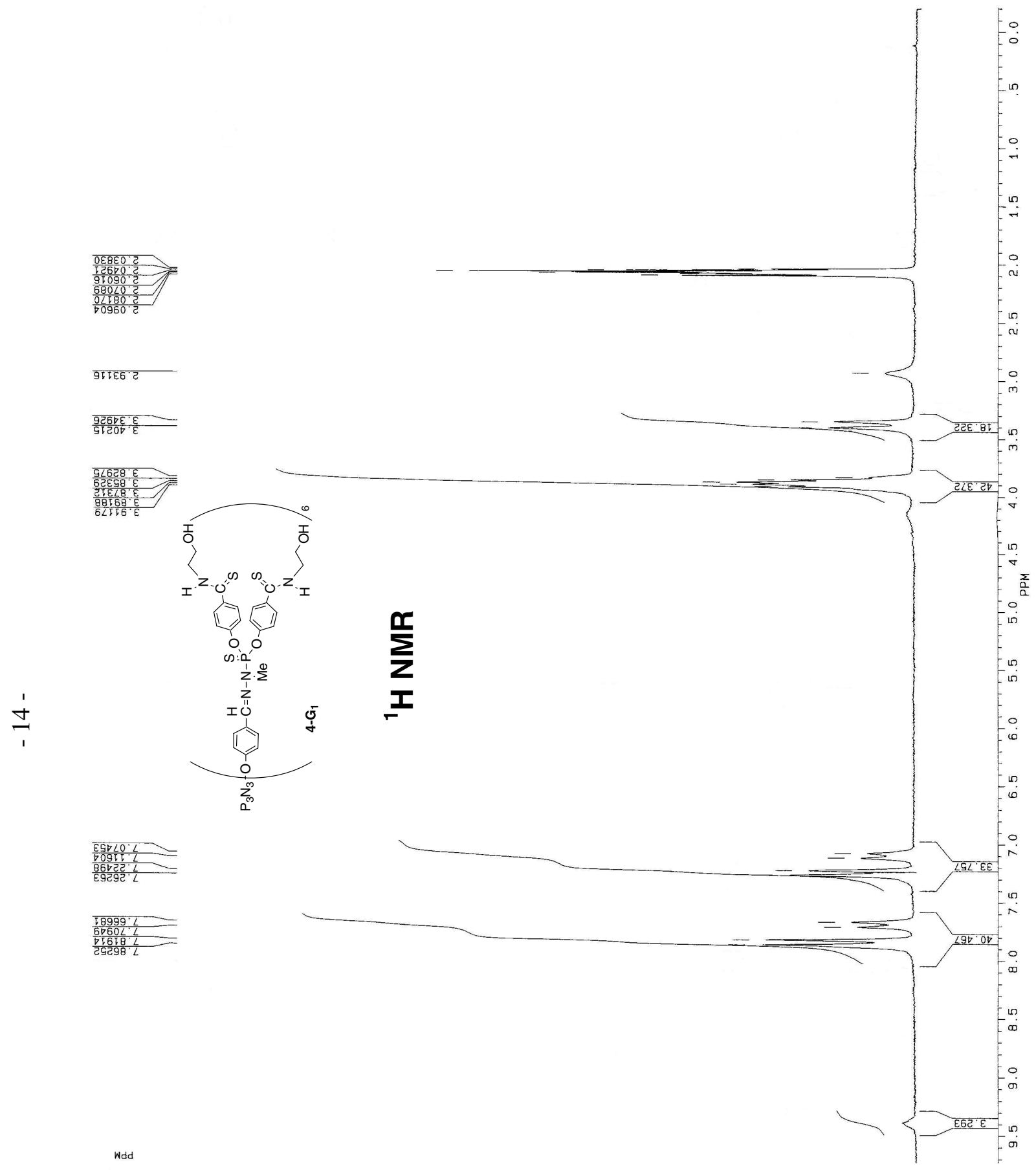



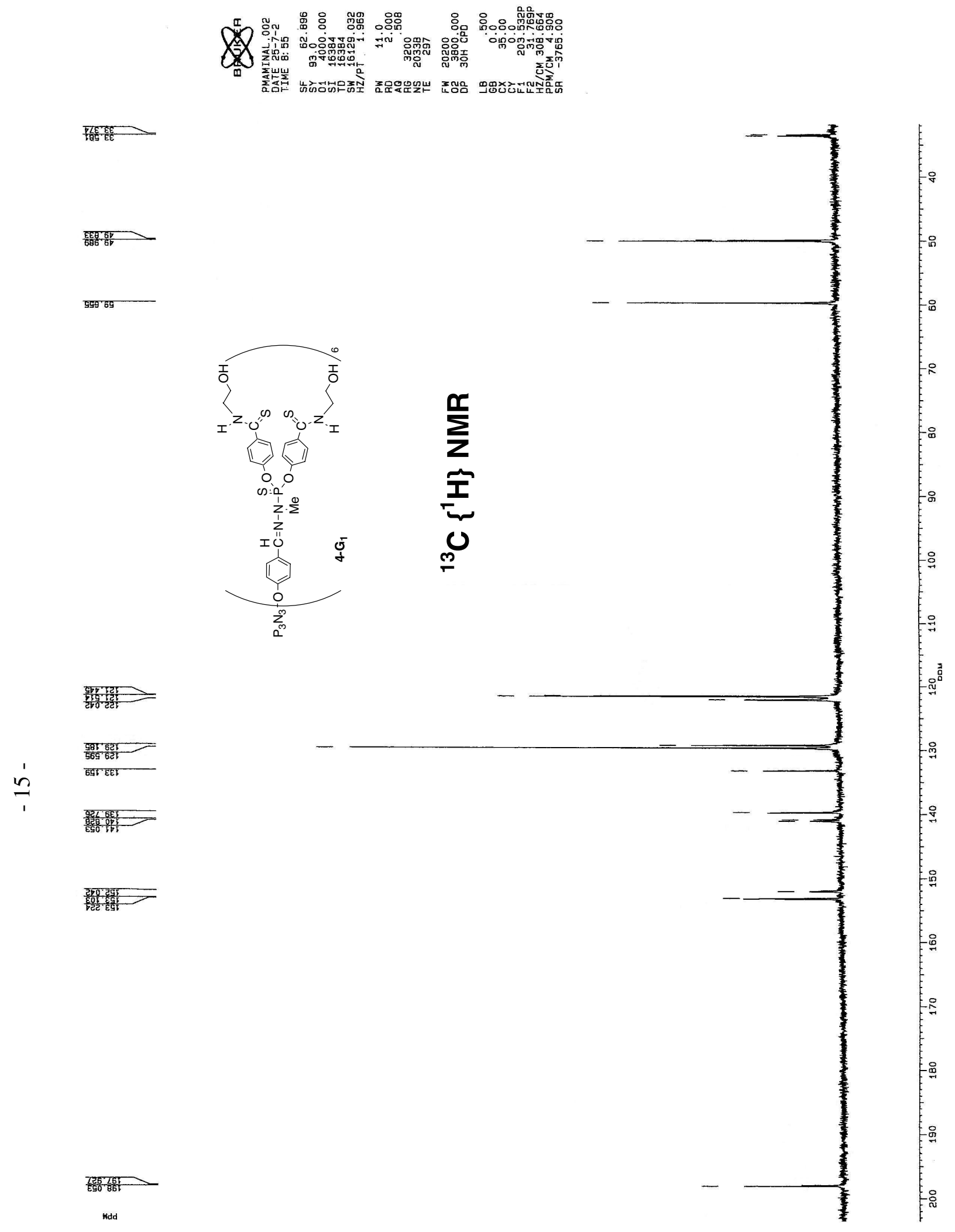

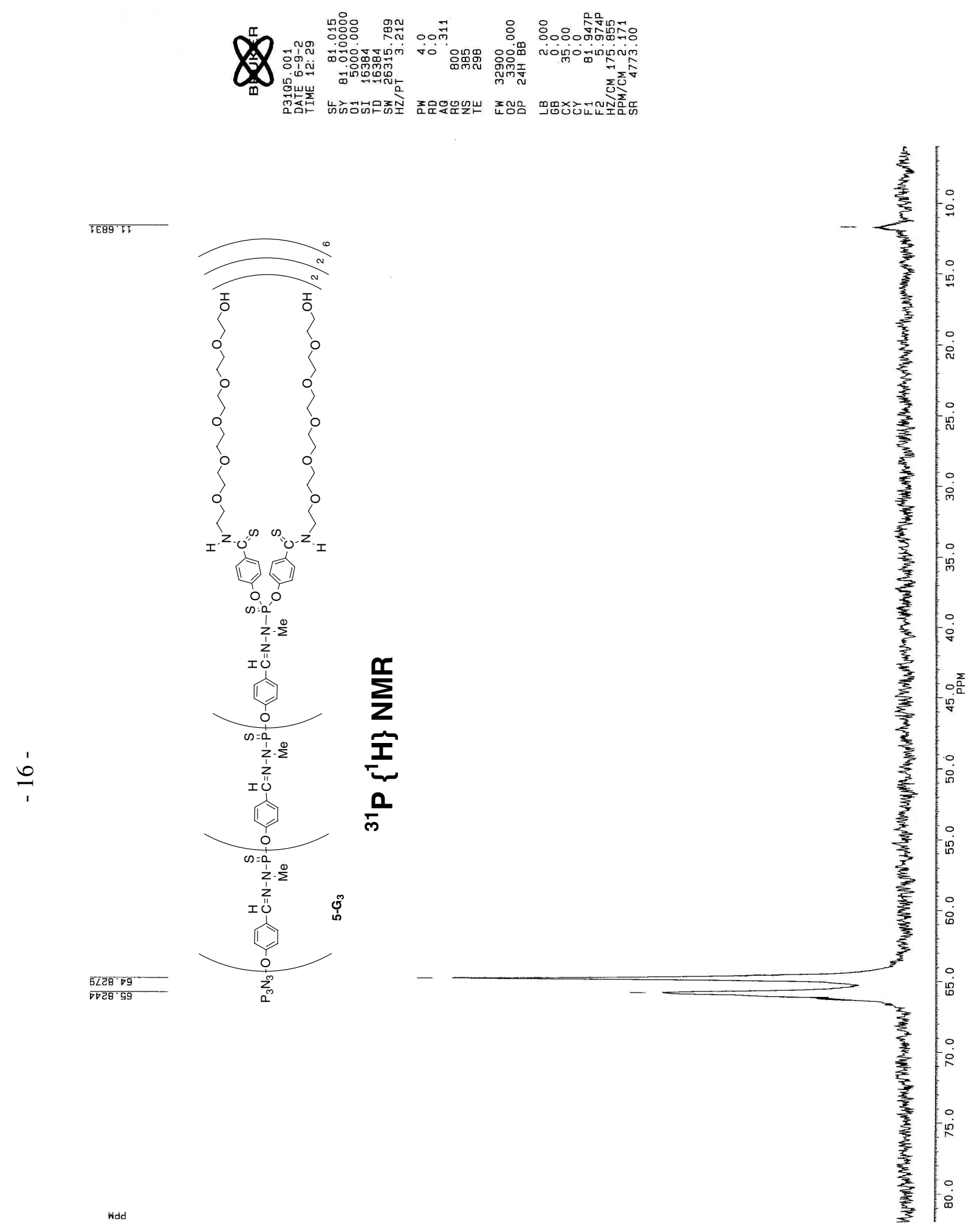


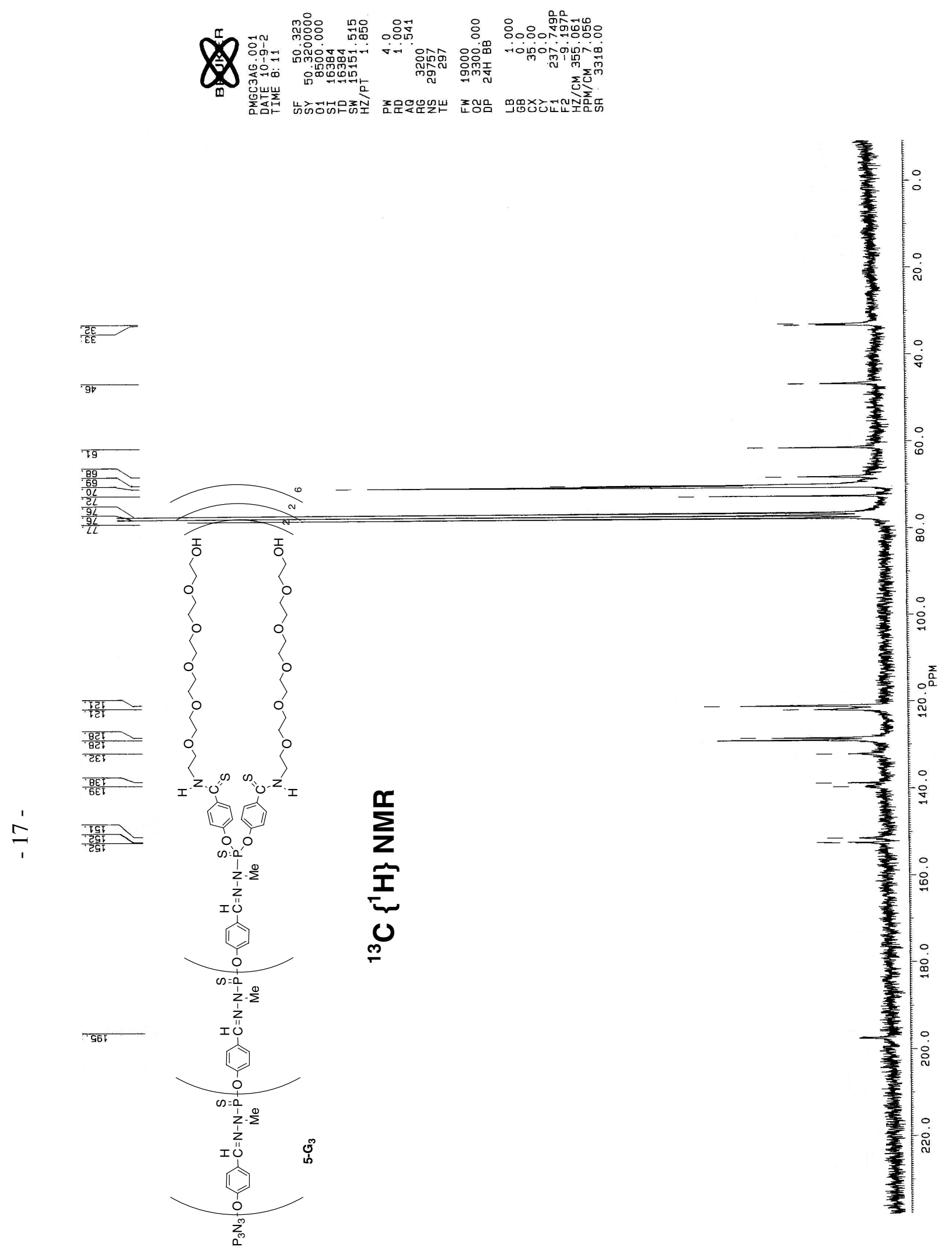



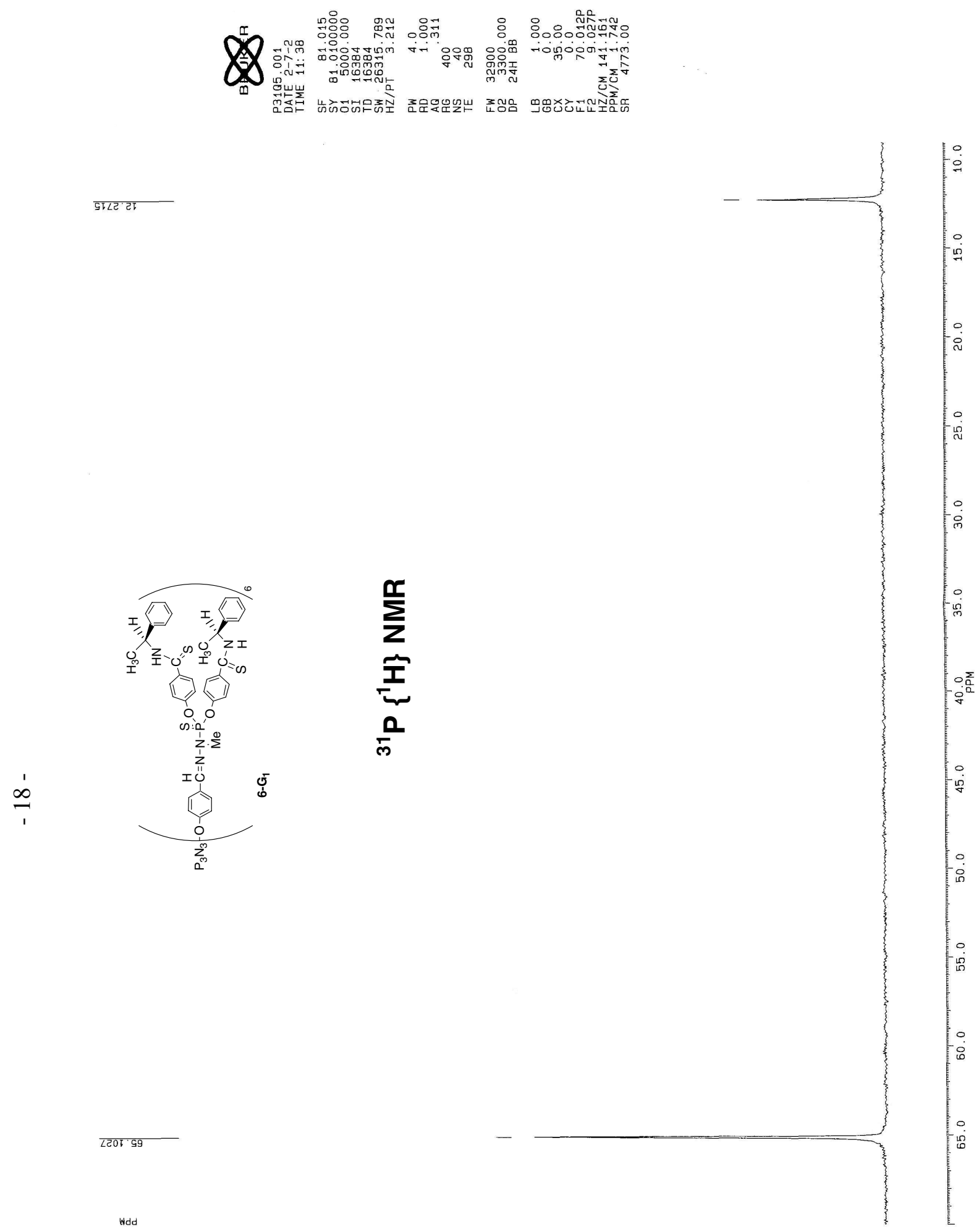

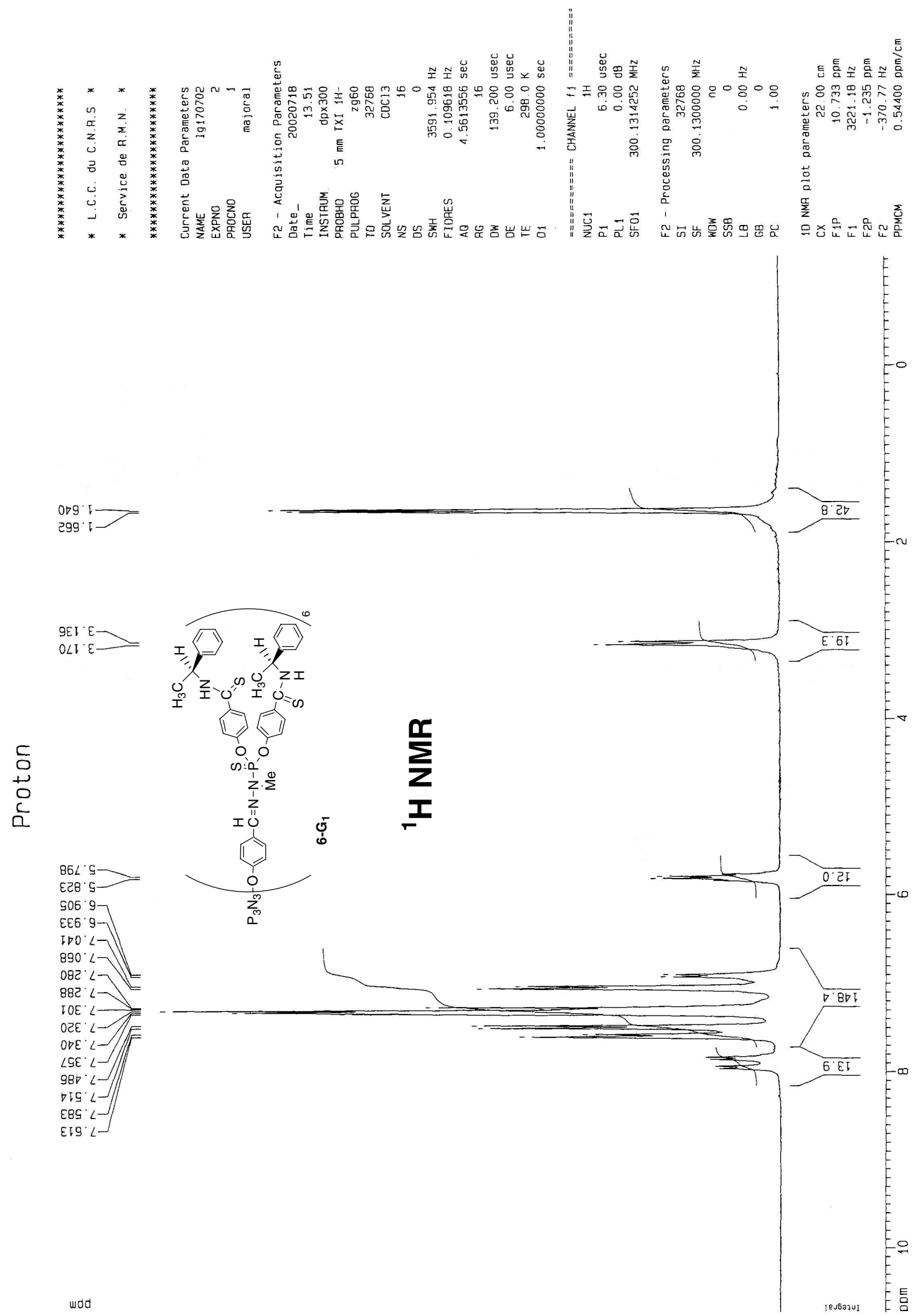


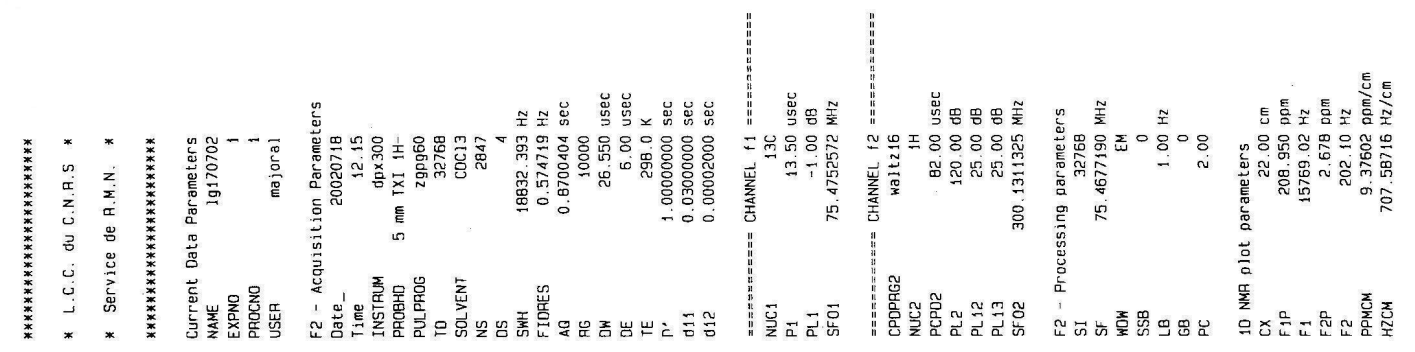

$9.02 \longrightarrow$
$8.05 \longrightarrow$

$\nabla E E \longrightarrow$
$9 \cdot E E \longrightarrow$

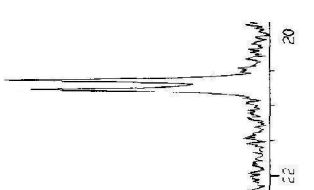

899
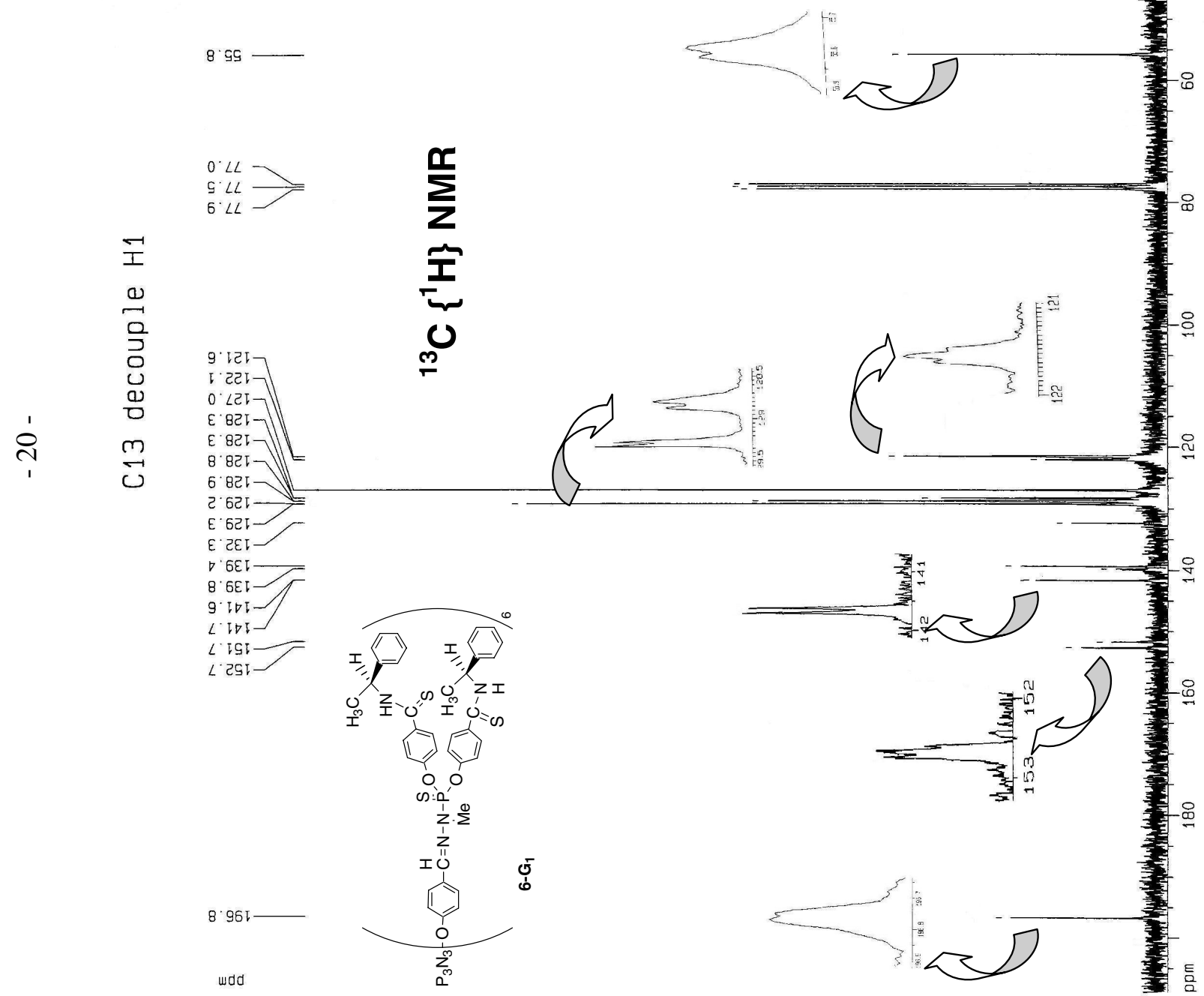